\title{
La Universidad y la formación permanente o la crónica de un fracaso del servicio público educativo
}

\author{
Miguel Ángel SENDín GARCíA \\ Universidad Europea Miguel de Cervantes \\ masendin@uemc.es
}

\begin{abstract}
Resumen
Uno de los elementos informadores de la nueva configuración de la formación universitaria es su consideración como un proceso de formación permanente. En este trabajo se analizan las motivaciones que subyacen bajo esta orientación que, en buena medida, conducen a una perdida sustantiva de derechos del trabajador y a un desequilibrio de la formación universitaria hacia la formación de profesionales, olvidando otros aspectos relevantes del sistema educativo. Se destaca en particular la falta de preocupación por dar una respuesta coherente a los colectivos más necesitados de esa formación permanente (desempleados de larga duración y jóvenes titulados que no han podido incorporarse al mercado laboral). Frente a ello choca la profunda atención que se ha prestado por el sistema universitario para rentabilizar estas necesidades de formación. Lo que demuestra el fracaso de las universidades en el cumplimiento de sus tareas como servicio público y su preocupante mercantilización.
\end{abstract}

Palabras clave: Universidad, Servicio público, formación permanente, sociedad de la información.

\section{University and constant education: Chronicle of an educational public service failure}

\begin{abstract}
Education as a constant process is one of the new university system inspiring ideas. This study analyses the real causes of this orientation. This conception involves a loss of worker's rights and shows a preference for one aspect of the university working system, professional training, leaving out other relevant elements. The lack of concern when it comes to giving a coherent response to the groups most in need of this training (the long-term unemployed and the young graduates who are unable to merge into the labour market) is particularly highlighted.

As opposed to this, the deep attention the University system has paid to make these training needs more profitable, looks, to some extent, shocking to us.

All of this is a good proof of the failure of universities in the fulfilment of its tasks as a public service and a sign of the University commercialization process.
\end{abstract}

Key words: University, public service, constant education, Information Society

\section{Referencia normalizada:}

Sendín García, M. A. (2013) La Universidad y la formación permanente o la crónica de un fracaso del servicio público educativo. Historia y Comunicación Social. Vol. $18 \mathrm{~N}^{\mathrm{o}}$ Especial Noviembre. Págs. 163-174.

Sumario: 1. Introducción. 2. Metodología empleada. 3. El nuevo mito de la formación permanente 4. La Universidad como servicio público. 5. El servicio público universitario ante las necesidades de formación permanente. 6. Las necesidades formativas en habilidades de comunicación. 7. Conclusiones 


\section{Introducción}

Muchos y profundos cambios ha experimentado este mundo que avanza ya decisivamente y sin pausa por el siglo XXI. El mundo de Internet y los móviles, del "WhatsAap" y los contratos de permanencia viaja, enarbolando la bandera del progreso técnico, superficial y a veces absurdo, contemplando impasible como millones de personas se mueren de hambre, enfermedad o inanición o sobreviven bajo condiciones infrahumanas, en condiciones no muy diferentes a las existentes hace 21 siglos.

Los que tenemos la fortuna de vivir en el mundo más avanzado nos complacemos, de forma un tanto infantil, en recalcar que nos hayamos en la "sociedad de la información", que comparte, pese a su nombre, con etapas históricos anteriores el gusto por la desinformación de lo que está pasando en esa otra parte del planeta menos agraciada, dejándonos vivir sin demasiadas quiebras de conciencia pendientes de nuestros videojuegos, reality show y otros inofensivos entretenimientos.

No todo es amable y luminoso, sin embargo, por más que los publicistas se esfuercen día a día en tratar de convencernos de lo contrario, en esta tierra bendecida por la Ciencia y el Progreso.

Esas disfunciones que durante mucho tiempo han pasado ocultas se están haciendo cada vez más manifiestas en los últimos años, en los que nuestro falso dios rey, "la Economía", nos acusa seriamente de pecar y nos llama a arrepentirnos: hemos sido, nos dicen, derrochadores y manirrotos y debemos hacer un esfuerzo de contención presupuestaria para expiar nuestros pecados.

De repente a la eterna tierra feliz de los demócratas se le ha perdido el cada vez más costoso calificativo de social y le empiezan a crecer los problemas. La crisis económica que con total falta de misericordia lleva llamando a nuestra puerta desde hace ya varios años, ha dibujado un panorama de pobreza y necesidad a la que no estamos ciertamente ya acostumbrados.

No es, sin embargo, en absoluto sorprendente, no es más que una crónica más de una muerte anunciada, pues los símbolos estaban ahí desde hace tiempo.

Se nos dice, no obstante, que no se trata de ninguna suerte de oculta conspiración, sino la simple consecuencia de las transformaciones de un nuevo tipo de sociedad, que dirigida por la siempre prudente mano del progreso técnico y científico exige una continua adaptación a los fulgurantes progresos tecnológicos que, supuestamente, nos hacen tan felices.

De este modo lo que toca ahora es adaptarse, ya no sirve de nada ni los años de experiencia, ni la intensa formación acumulada a lo largo de años de esfuerzo, ni la fidelidad a la empresa, todos somos trabajadores fácilmente intercambiables, cuya principal virtud debe ser la de sabernos adaptar a las novedades.

Un discurso con lengua de serpiente que se ha llevado de un plumazo nuestros derechos sociales y laborales, ya no hay convenio colectivo que valga, ni condiciones más favorables, los agentes sociales se deben cuadrar ante las exigencias presupues- 
tarias, o la cosa puede ir incluso a peor, pues nos pueden traer a esa nueva versión del hombre del saco que llaman deslocalización, que amenaza con llevarse nuestros puestos de trabajo a un país sin derechos, donde los explotadores puedan campar a sus anchas.

La educación, claro está, no podía quedar al margen de esta evolución y así, de repente, deja de ser esa actividad casi sagrada dirigida a la formación del individuo para lograr su realización personal y social, y se convierte en una suerte de formación profesional, que transforma nuestras universidades en una obstinada fabrica encargada de producir acríticos profesionales para los que, no nos engañemos, en muchos casos, por no decir en la mayoría de los casos, no hay trabajos.

No se nos entienda mal, no queremos negar una realidad difícilmente cuestionable como lo es la mayor importancia que una formación a lo largo de la vida puede tener en el presente contexto social. Lo que queremos criticar es el considerable grado de cinismo con que se está utilizando ese hecho cierto para la consecución de otros fines no tan ciertos.

La importancia de la formación permanente es innegable en cuanto constituye un auténtico bien que todos tienen derecho a disfrutar y, por lo tanto, hacer uso de la misma en cualquier momento vital es un derecho incuestionable.

No menos evidente es la especial importancia que está cobrando en un contexto como en el actual en el que los cambios sociales y tecnológicos se han hecho especialmente veloces, generando notables lagunas no ya sólo en el personal más antiguo, sino, en realidad, prácticamente en la totalidad de los trabajadores.

No queremos negar la importancia de ofrecer una formación que supla estas deficiencias formativas, declaramos clara y directamente su trascendental importancia. Lo que queremos es cuestionar es la veracidad de un discurso bífido, que emplea falsa y maliciosamente unas causas y justificaciones para fundamentar decisiones que no tienen nada que ver con ellas y que pretenden unos objetivos muy diferentes. Siempre se ha dicho que no hay más mentiroso que el que dice medias verdades y eso es a lo que nos enfrentamos, a unas verdaderas utilizadas ladinamente para dar legitimación a decisiones que se toman por causas muy diferentes, en ocasiones, incluso, puramente contradictorias a las declaradas.

\section{Metodología empleada}

No podíamos llevar a cabo esta tarea siguiendo los pasos de los comentarios legislativos tradicionales, limitados a hacer un conjunto de observaciones, en algunos casos de forma crítica, sobre los textos normativos empleados. Nuestro trabajo trata más bien de desentrañar cuales son las motivaciones reales que subyacen bajo la normativa y declaraciones políticas comentadas. 
Se ha prestado, por tanto, especial atención al contexto real de los problemas a los que presuntamente se enfrenta la normativa y textos programáticos afectados, determinando si la realidad que esos documentos y normas proclaman coinciden con la real y si las soluciones arbitradas por las normas legales vigentes es adecuada para darle solución a las mismas.

Partiendo de las contradicciones que nos ha arrojado el contraste de ese substrato real con la normativa analizada se ha tratado de identificar las motivaciones reales de esas medidas políticas y legislativas cuando se ha descubierto una desviación, lo que nos ha permitido hacer un juicio crítico, en el que se concluye que se está realizando una inadecuada utilización de determinados problemas y realidades sociales para justificar medidas que no pretenden resolver esos problemas, sino buscar unos objetivos diferentes.

\section{El nuevo mito de la formación permanente}

Uno de los principales elementos sobre los que se ha construido el nuevo entramado universitario bajo la inspiración del Espacio Europeo de Educación Superior (EEES) ha sido la consideración de la Educación como un proceso de formación permanente que se desarrolla a lo largo de toda la vida (González García, 2009: 19).

En términos muy sencillos esto viene a suponer que ya no se distinguen en el desarrollo del individuo dos etapas netamente separadas: la de estudiante, que comprendería hasta el fin de sus estudios universitarios, incluyendo en su caso la realización de un master y/o doctorado; y la profesional, que se desarrollaría una vez terminados los estudios universitarios e incorporado a la vida profesional.

Ahora se entiende que la etapa como estudiante no acaba nunca, pues el mundo dinámico en el que vivimos requiere de una constante adaptación del trabajador a los nuevos acontecimientos, sin la cual se deja de ser competitivo.

De este modo la actualización del trabajador deja de ser una especie de elemento de mejora, dirigido a su realización personal y a una posible promoción respecto a su puesto de trabajo, para convertirse, en gran medida, en un elemento necesario para conservar el puesto de trabajo.

A la vez, esa actualización se convierte en un factor decisivo para la competitividad tanto de la empresa como del propio país, o en un sentido más adecuado a nuestro contexto económico actual, de la Unión Europea, sujeto que ha jugado un papel muy activo en la difusión de esta forma de pensar a través de la consolidación del EEES (Consejo de Universidades, 2010: 13-14).

No han faltado, no podía ser de otro modo, las voces críticas con este discurso, que inciden especialmente en la consideración de que esta forma de pensar constituye una 
relevante economización de algo que debe estar más allá de las condiciones económicas, como es la educación.

Desde este pensamiento crítico se destaca como el fenómeno educativo se está orillando peligrosamente hacia uno de sus componentes, la formación profesional, dejando de lado otros aspectos igual de relevantes o más, como son el desarrollo personal del individuo y la formación de una masa crítica de ciudadanos (Murga Menoyo y Quicios García, 2006: 9).

La respuesta vertida desde los partidarios de la formación permanente a este sector crítico descansa en razones de hecho, no es, entienden, una actitud política, social o económica, sino una simple respuesta a los nuevos presupuestos sociales en que nos sitúa la llamada sociedad de la información (Buxarrais, 2006: 39).

Esta discusión nos sitúa, creemos, ante una cuestión de principios, que sólo puede resolverse partiendo de un elemento medular, como lo es el del propio papel que la universidad debe jugar. Eso, nos obliga, nos parece, a realizar una profunda reflexión sobre la esencia misma de la institución universitaria.

\section{La universidad como servicio público}

Pocas expresiones hay tan polisémicas y contradictorias como la de "servicio público". Esto hace obligado, si se quiere operar con la más mínima seguridad jurídica, aclarar con qué sentido se está utilizando el término.

Perjudicado por una sesuda reflexión doctrinal, muy marcada además por tintes ideológicos, el término "servicio público" se ha convertido en un concepto considerablemente confuso, a la par que en un clásico del discurso político y jurídico (Sendín García, 2003: in totum y Sendín García, 2006: in totum).

Para hacer más sencillas las cosas, aunque suponga, somos conscientes de ello, una notable simplificación, nos vamos a quedar con los dos significados más recurrentes con los que se suele identificar.

El primero de ellos, sin entrar en más precisiones, que se comerían este breve estudio, es el que vincula nuestro término con la prestación pública en alguna de sus formas. Baste como ejemplo las muy de moda, la crisis lo ha hecho inevitable, expresiones del tipo "salvemos el servicio público educativo" o "protejamos la consideración de la Sanidad como un servicio público". En ambos casos, se entiende el servicio público como una actividad prestada por los poderes públicos. No es ni mucho menos el sentido con el que se utiliza en este estudio.

El segundo significado, quizás menos técnico y más propio del lenguaje político, a pesar de lo cual es con el que nos queremos quedar en este trabajo, entiende por servicio público aquella actividad que se presta no en atención a intereses particulares y egoístas, sino para el bien común. 
En tal sentido, la universidad es un servicio público por los valores a los que sirve, es una institución al servicio de la sociedad, a cuya disposición pone a la ciencia y el conocimiento.

\section{El servicio público universitario ante las necesidades de formación perma- nente}

Ahora bien, ¿invalida esto una perspectiva como la que hemos visto se impone en los últimos años, que toma la formación para el desarrollo profesional y la competitividad empresarial como referencia? En nuestra opinión no, la formación de profesionales altamente cualificados es uno de los pilares estructurales sobre los que debe construirse la Universidad. Si bien, no el único, la formación de un alumnado crítico $\mathrm{y}$, en general el fomento del conocimiento como un fin en sí mismo, con independencia de su utilidad para un eventual desarrollo laboral, no son menos importantes.

Igualmente es innegable que ese servicio a la colectividad se debe adaptar a las necesidades sociales imperantes en cada momento y, hoy en día es incontrovertible que la búsqueda de un profesional con capacidad para adaptarse a los continuos cambios técnicos y del mercado es una tarea ineludible.

La cuestión parece entonces clara, si partimos de la consideración de que la Universidad está llamada a dar satisfacción, entre otras cuestiones, a las necesidades de formación a lo largo de toda la vida que nos plantea la nueva situación social, debemos preguntarnos si ha emprendido esa tarea con arreglo a las exigencias que se deriva de su consideración como un servicio público.

Nos parece obvio que nuestra educación superior sólo se puede considerar que está a la altura de tan alto cometido si toma las necesidades de los ciudadanos como primer elemento informador, tomándolo como un elemento preferente a las siempre relevantes consideraciones económicas.

Sentimos comunicar en dicho sentido que dicha batalla ha concluido, siempre según nuestra opinión, con una severa derrota del interés público ante el interés comercial. Esta realidad que quizás podría haber pasado con disimulo, ocultando su rostro bajo otras ropajes, se ha visto descubierta por las graves consecuencias de la crisis económica a la que asistimos desde hace ya demasiados años.

Desgraciadamente esa funesta quiebra del equilibrio económico de nuestras sociedades, que ahora nos dicen, ya veremos en que queda, estamos a punto de superar, va a dejar, aún en el mejor de los casos (esto es, en el supuesto de que verdaderamente estemos llegando al final de ese largo túnel, cosa que no nos creeremos hasta que lo veamos), un reguero de victimas que, como daños colaterales de una guerra que no era la suya, se van a encontrar al borde de un abismo de exclusión social del que les va a ser más que difícil salir. 
El grupo más preocupante es, obviamente, el de aquellos ciudadanos que salen de una situación de paro de larga duración y se encuentran además en edades avanzadas. Un colectivo al que le va a ser especialmente difícil acceder a un cada vez más exigente mercado laboral (las últimas noticias de los telediarios nos dicen que algunos ayuntamientos piden ahora un curriculum hasta para ser cantante callejero) y que se pueden ver especialmente perjudicados por la tan cacareada dinamicidad del mercado laboral, pues ya no sólo sus estudios, sino también su propia experiencia laboral se ha quedado desfasada.

Tampoco están en posición de celebrar muchas fiestas los nuevos titulados. Una hornada de nuevos profesionales, algunos de ellos como no dotados de gran talento $\mathrm{y}$, probablemente, la generación mejor formada de nuestro país, que van a estrellarse con una realidad de puertas cerradas que va a convertir sus ilusiones de acceder al mercado laboral en muchos casos en eso, simples ilusiones.

Aquellos que no sucumban al desaliento y pretendan resistir a esa injusta realidad que les va a obligar a conformarse con puestos inadecuados a su capacidad o a coger sus maletas para ir el extranjero (y en muchos casos a ambas cosas, pues fuera de nuestro país tampoco se atan los perros con longanizas) deberán unirse a sus compañeros desempleados de edad avanzada y continuar reforzando su formación.

Choca sobremanera la falta de sensibilidad de los integristas de la formación continua a esta dura realidad, sin duda la más importante manifestación de su necesidad, a la par que la más dramática. No sobran, sin embargo, aquí las tentativas para la resolución de este problema. No vemos a las autoridades educativas (ya sean estatales, autonómicas o europeas) especialmente preocupadas por este drama humano.

Más bien pareciera que la formación permanente fuera sólo una obligación para aquellos que tienen un puesto de trabajo, que se pueden ver tachados de incompetentes en cualquier momento por no haberse adaptado no ya a las nuevas técnicas y tecnologías de su profesión, sino también a las nuevas modas u ocurrencias gerenciales de turno, que pueden decidir en cualquier momento que tu empresa de toda la vida va a diversificar su actividad va a dedicarse, por ejemplo, a vender pizzas, y que tu dejas tus, por poner un caso, actividades contables para dedicarte a cocinar estos suculentos manjares.

En algún lugar del camino se han quedado perdidas y olvidadas no sólo la experiencia laboral y los servicios prestados sino, lo que es más grave, los derechos mínimos de los trabajadores conseguidos como consecuencia de largos años de lucha.

Me comentaba el otro día una compañera de trabajo que vivimos en una época en la que se trabaja con las maletas en la mano, pues en cualquier momento te puedes ver forzado a cambiar de empresa o de puesto de trabajo.

El trasfondo de esa realidad incontrovertible no es en absoluto amable, pues esconde una considerable dosis de sufrimiento. Nuestro moderno y bienintencionado estado social ofrece si se le examina con ojos objetivos un pobre juicio. No hay que poner mucha atención para ver un reguero de familias desagregadas, que se ven peno- 
samente los fines de semana, con suerte, de niños de demasiada guardería y de padres condenados a una vejez de residencia.

En cierto modo parece que nos estuviéramos conduciendo penosamente a los crueles postulados de una educación espartana en los que los hijos dejan de ser nuestros para ser cada vez más de toda la sociedad, pues no es sinceramente que los veamos mucho y no precisamente porque no queramos estar con ellos.

Parece, pues, que la ideología de la educación permanente no piensa tanto en la necesidad de resolver el difícil problema de un amplio colectivo de trabajadores que la necesitan para no caer aún más en la exclusión y que, probablemente, no se la van a poder pagar por sus propios medios.

Lo relevante es más bien dejarnos claro a todos que nuestras licenciaturas o grados, másteres y doctorados y años de experiencia no nos salvan de una eterna consideración de becario, al que se puede seguir exigiendo en cualquier momento que deje sus tareas y se dedique a traer cafés.

No se agota, evidentemente, aquí el recorrido de la formación a lo largo de toda la vida, los mercados que nos mandan no han podido dejar de ver que esa ingente masa de desempleados (que incluye los que están declarados formalmente como tales y los disimulados bajo el disfraz de eterno estudiante, que continúa estudiando grados, másteres, doctorados u otro tipo de cursos para seguir con persistencia infinita preparándose para un trabajo que nunca llega) constituye un volumen de negocio considerable, que convierte el sector educativo en uno de los más dinámicos del momento actual. Hay dinero en el ambiente y esto sí que se huele rápido.

No han faltado, por ello, iniciativas animosas para pescar en este río revuelto y captar ávidamente la parte de un botín que alimenta la desesperación de gran número de familias que se gastan unos ahorros que en el mejor de los casos necesitan para otras cosas y, en el peor de los casos, simplemente no tienen, en un último intento desesperado por proporcionar al ser amado (hijo, esposo, hermano...) un puesto de trabajo que le salve de la desesperación personal y la amenaza de caer en la pobreza.

Es en este punto, si estuviéramos en un película comercial americana, es donde debería aparecer el héroe salvador que, en nuestro contexto, no nos engañemos, no puede ser otro que la Administración pública, que debería extender su mano generosa, en este caso sobre todo en forma de Universidad para poner un poco de cordura en este desaguisado social y educativo.

Nuestro defensor de lo justo no ha llegado, sin embargo, y lo que es peor aún, no se lo espera, pues no parece que de otra forma se pueda entender la rotunda subida de tarifas que las universidades están aplicando a sus servicios en general y, más particularmente, a los de postgrado (master y doctorado) de remarcable importancia en la materia que nos ocupa.

No menos preocupante es la total falta de adaptación de las titulaciones ofrecidas por el sistema universitario, muy alejadas de la realidad que demanda el mercado de 
trabajo y, en muchas ocasiones, un simple agregado de formaciones, que son casi siempre además, una repetición más o menos literal de la formación que ya se ha impartido al alumno durante el grado. Un mero entretenimiento, que retrasa la incorporación del alumno al mercado de trabajo, o lo que es más preciso a las listas del paro, aliviando con ello las urgencias estadísticas del gobernante de turno, sin aportar por lo demás gran cosa a la formación del alumno. Aunque eso sí, aligerando notable e indefectiblemente sus bolsillos o los de sus progenitores.

Somos muchos los que pensamos que, con ello, los mercaderes han vuelto a entrar en el templo, pues eso es lo que es, en cierto sentido, la educación superior, un espacio que debiera estar inspirado por valores y principios más altos que los aires contables que ahora lo invaden todo, un espacio para la realización personal y social del individuo, un espacio para poner la ciencia y el progreso a disposición del interés de todos. En definitiva, un servicio público.

Si nuestro admirado Duguit que tanto lucho por difundir la idea de servicio público, a la que imprimió un carácter social que se ha quedado adherido a su piel, inseparable ya del mismo (Rodríguez-Arana Muñoz y Sendín García, 2010: 51 y ss.), levantase la cabeza, volvería raudo y veloz al mundo de los muertos, derrotado y abochornado por el fracaso de su sueño.

\section{Las necesidades formativas en habilidades de comunicación}

Si hay un ámbito, por lo demás, en el que esta evolución alcanza su grado más alto es, sin duda, el de las competencias comunicativas, en dos vertientes. Por un lado, la capacidad para hacer un uso adecuado de las nuevas tecnologías, que constituyen hoy un elemento estructural para la competitividad de cualquier empresa, y, por otro lado la capacidad para comunicarse en lenguas extranjeras, esencialmente en inglés.

En cuanto al primer aspecto, la necesidad de formación permanente se hace presente por dos grandes motivos. En primer lugar, porque la propia celeridad de los cambios tecnológicos determinan que todos los trabajadores queden continuamente desactualizados.

En segundo lugar, por la existencia de amplias capas de la sociedad cuya formación y experiencia profesional se gestó durante un tiempo en que esas tecnologías aún no existían y necesitan de una decidida intervención educativa para adaptarse a los nuevos tiempos y métodos.

En lo que se refiere al segundo aspecto, en un mundo tan internacionalizado como el que vivimos hoy, tener una capacidad de comunicarse en otros idiomas, en particular en inglés, resulta prácticamente imprescindible para un elevado número de trabajos. Existiendo, sin embargo, un gran número de ciudadanos que carecen de esas habilidades. Problema especialmente urgente en nuestro país por las tradicionales deficiencias en la formación en esta materia que hemos sufrido desde siempre. A 
lo que se añade el elevado número de personas que, en su momento, recibieron la formación de segundo idioma en lengua francesa.

Ni que decir tiene que estas deficiencias se hacen más urgentes en los colectivos desfavorecidos a los que antes nos referimos, desempleados de larga duración y jóvenes titulados demandantes de su primer trabajo. La pasividad del servicio público universitario ante esta problemática resulta, una vez más, sorprendente.

\section{Conclusiones}

Nos resulta ante todo lo dicho difícil situarnos ante la penosa tarea de sintetizar la poca agradable realidad narrada en este artículo en unas breves conclusiones, sintéticas y precisas, como exige el rigor académico, sobre todo porque nada es más crudo que la verdad desnuda y ésta nos arroja el siguiente resultado, de difícil digestión, al menos para aquellos que decidimos dedicarnos a la docencia e investigación pensando que a la vez que una profesión estábamos adquiriendo la posibilidad de hacer algo por la colectividad.

En fin, no nos perdamos en consideraciones y elucubraciones y enumeremos sin más dilaciones nuestras conclusiones despejadas de artificio, lo que nos lleva a afirmar que:

$1^{\circ}$. El discurso de la formación permanente encubre un desapoderamiento considerable de los derechos del trabajador, que pasa a una situación de total inestabilidad al acuñarse el mito de la preponderancia de la adaptación a las nuevas necesidades sobre los años de formación y experiencia.

$2^{\circ}$. Esa inestabilidad laboral impide la posibilidad de conciliación de la vida laboral y familiar, que simplemente no existe para la mayor parte de la población, que vive continuamente bajo la amenaza de perder su puesto de trabajo. Viéndose, en consecuencia, obligado a asumir modificaciones de su puesto de trabajo o perfil profesional que merman notablemente su desarrollo profesional y su nivel de vida.

$3^{\circ}$. Existe un número considerable de ciudadanos que han perdido su puesto de trabajo a edades avanzadas y que en su mayor parte si no se toman medidas drásticas caerán en una situación definitiva de exclusión social.

$4^{\circ}$. Los jóvenes licenciados/graduados carecen en su mayoría de posibilidades de acceso a puestos de trabajo de su capacidad. La probabilidad de acceder a esos puestos va a ser menor a medida que pase el tiempo si no consiguen acceder a un primer empleo que les dote de experiencia, pues carecen de esa experiencia previa y se alejan de las condiciones para acceder a un contrato de becario o "contrato basura" que es en la mayor parte de los casos la única vía para acceder a su profesión.

$5^{\circ}$. Una de las pocas opciones de salida que tienen esos colectivos es continuar formándose. 
$6^{\circ}$. El sistema educativo y, en especial el universitario, no sólo no da una respuesta adecuada a estos colectivos, sino que ha respondido aprovechando la oportunidad de negocio que eso supone, convirtiendo la educación superior en un asunto de mercaderes que ofrece títulos educativos, en muchos ocasiones de dudosa utilidad, y a un precio excesivamente caro y creciente, aprovechándose de la desesperación de las familias que tienen medios económicos y un familiar en situación de desempleo, que están dispuestas a hacer cualquier cosa por darles una posibilidad.

$7^{\circ}$. En el ámbito de las competencias de comunicación está realidad se hace especialmente presente, dada las importancia de tener una alta capacidad en el manejo de las nuevas tecnologías y en el dominio de lenguas extranjeras, en particular del inglés, para acceder al mercado de trabajo.

$8^{\circ}$. No nos gusta esta realidad, es más nos abochorna. Más aún cuando sentimos que como profesores no dejamos de formar parte de esa realidad amarga y oscura. No podemos dejar, por tanto, las cosas aquí y reivindicamos desde este foro la importancia de la labor universitaria. Proclamamos, en consecuencia, la necesidad de corregir esta oscura evolución que nos conduce a una realidad injusta y socialmente desigual, exhortando a nuestros responsables educativos a conducir a nuestro sistema universitario por el camino que nunca debió abandonar: el del servicio público.

\section{Bibliografía}

BUXARRAIS, M. R: "La misión de la Universidad en la construcción de la ciudadanía europea”, en MURGA MENOYO, M. A. y QUICIOS GARCÍA, M. del P. (Coordinadoras): La reforma de la Universidad. Dykinson. Madrid.

CONSEJO DE UNIVERSIDADES (2010): La formación permanente y las Universidades españolas. Comisión de Formación Continua, disponible en http://www. mecd.gob.es/dctm/eu2015/2010-formacion-permanente-universidades-espanolas-060710.pdf?documentId=0901e72b802bcfbf [fecha de consulta 17/07/2013].

GONZÁLEZ GARCÍA, J. V. (2009): "Introducción”, en GONZÁLEZ GARCÍA, J. V. (director): Comentario a la Ley Orgánica de Universidades. Thomson/Reuters. Navarra.

MURGA MENOYO, M. A. y QUICIOS GARCÍA, M. DEL P. (2006): "Presentación", en MURGA MENOYO, M. A. y QUICIOS GARCÍA, M. del P. (Coordinadoras): La reforma de la Universidad. Dykinson. Madrid.

RODRÍGUEZ-ARANA MUÑOZ, J. y SENDÍN GARCÍA, M. Á. (2010): "El servicio público según Duguit ¿una referencia para un mundo en crisis? En: Revista Jurídica de Canarias $\mathrm{n}^{\mathrm{o}}$. 18.

SENDÍN GARCÍA, M. Á. (2003): Hacia un servicio público europeo. El nuevo Derecho de los servicios públicos. Comares. Granada.

SENDÍN GARCÍA, M. A. (2006): “La educación como servicio público”. En: Diario La Ley 6482. 


\section{El autor}

Miguel Ángel Sendín García es Profesor de la Universidad Europea Miguel de Cervantes, dónde fue Vicerrector de Ordenación Académica y Profesorado. Acreditado como Profesor Titular de Universidad. 